\title{
The Core of Roommate Problems: Size and Rank-Fairness within Matched Pairs*
}

\author{
Paula Jaramillo ${ }^{\dagger}$ Çağatay Kayı ${ }^{\ddagger}$ Flip Klijn ${ }^{\S}$
}

September 15, 2018

\begin{abstract}
This paper deals with roommate problems (Gale and Shapley, 1962) that are solvable, i.e., have a non-empty core (set of stable matchings). We study rank-fairness within pairs of stable matchings and the size of the core by means of maximal and average rank gaps. We provide upper bounds in terms of maximal and average disagreements in the agents' rankings. Finally, we show that most of our bounds are tight.
\end{abstract}

Keywords: matching, roommate problem, stability, core, rank-fairness, rank gap, bound.

JEL-Numbers: C78.

\section{Introduction}

In roommate problems (Gale and Shapley, 1962), a group of agents wish to divide up into pairs and singletons. Each agent has a preference list or so-called ranking over the other agents and being single (which can be interpreted as recurring to an outside option). The central solution concept for roommate problems is the set of stable matchings (or core), i.e., the partitions of agents in pairs and singletons for which there are no blocking coalitions.

${ }^{*}$ We thank David Manlove for useful suggestions on the computation of stable matchings for roommate problems. We thank Bettina Klaus, Fuhito Kojima, Andreu Mas-Colell, and seminar audiences at Universitat de Barcelona and Universitat Rovira i Virgili for useful discussions. We thank an Associate Editor and three anonymous reviewers for their valuable comments and suggestions.

†Universidad de Los Andes, Bogotá, Colombia.

$¥$ Universidad del Rosario, Bogotá, Colombia.

${ }^{\S}$ Corresponding author. Institute for Economic Analysis (CSIC) and Barcelona GSE, Spain; e-mail: flip.klijn@iae.csic.es. The first draft of this paper was written while F. Klijn was visiting Universidad del Rosario. He gratefully acknowledges the hospitality of Universidad del Rosario and support from AGAUR-Generalitat de Catalunya (2014-SGR-1064 and 2017-SGR-1359), the Spanish Ministry of Economy and Competitiveness through Plan Estatal de Investigación Científica y Técnica y de Innovación 2013-2016 (ECO2014-59302-P and ECO2017-88130-P), and the Severo Ochoa Programme for Centres of Excellence in R\&D (SEV-2015-0563). 
OBSERVATION: If all agents' rankings are based on a (common) ordering of the agents, ${ }^{1}$ then there is a unique stable matching that is, moreover, rank-fair (within matched pairs).

Rank-fairness refers to the resemblance of the "ranks" (i.e., positions in the rankings) that the two members of any matched pair assign to one another. A stable matching is rank-fair (within matched pairs) if the "rank gap" in each pair of matched agents is nil, i.e., its two members assign the same rank to one another. In other words, no agent can argue that his mate is treated more favorably. Obviously, this is a relatively weak form of fairness: there are no comparisons with agents different from his mate. ${ }^{2}$

The observation above applies to real-life applications when there is a criterion according to which the agents order themselves. For instance, in case of roommates that have to share rooms in dorms or police-officers that have to go on patrol in couples, this criterion could be seniority. However, it is not unlikely that there are multiple criteria and that preferences vary from one agent to another. What can we say about the core in this more realistic case?

A first problematic issue in tackling this question could be the possible non-existence of stable matchings. ${ }^{3}$ However, Chung (2000) provides a number of sufficient conditions for the existence of a stable matching that are economically interpretable. ${ }^{4}$ Hence, in view of Chung's (2000) findings it seems reasonable to not worry (too much) about the non-existence but focus instead on the robustness of the observation above. More specifically, our results show that if rankings are "sufficiently similar" then (i) no stable matching can be very "rank-unfair" and (ii) the "size" of the core cannot be very large. Next, we explain in more detail our results.

To formally state our results, we define (dis)similarity of rankings in terms of "disagreements" over agents. The disagreement over an agent is the difference between the maximal and the minimal rank of the agent in the other agents' rankings. Section 3 delivers two results (Theorems 1 and 2) that show (i). We say that a stable matching is not very rank-unfair if maximal/average ${ }^{5}$ rank gaps are "small." Theorem 1 provides an upper bound of the maximal

\footnotetext{
${ }^{1}$ More precisely (and without loss of generality), if the set of agents is $N=\{1, \ldots, n\}$, then agent $i$ 's ranking is (from most to least preferred) $1,2, \ldots, i-1, i+1, \ldots, n-1, n$ (being unmatched is the worst option).

${ }^{2}$ Klaus and Klijn (2010) do make comparisons between all agents, but they only consider "weak rankings" that contain stable mates (i.e., partners obtained at stable matchings). More precisely, let $S$ be a set of $2 k+1$ stable matchings. For each agent $i$ consider the "weak ranking" that is obtained by restricting his original ranking to mates obtained in $S$ and such that if he is matched to some agent $j$ at exactly $l$ stable matchings in $S$ then $j$ is listed $l$ times. Then, assigning each agent to his $(k+1)$-st (weakly) most preferred mate in the weak ranking constitutes a stable matching (Klaus and Klijn, 2010, Theorem 2). Klaus and Klijn (2010, Corollary 1) deals with the case of a set with an even number of stable matchings.

${ }^{3}$ Gale and Shapley (1962) exhibit an unsolvable roommate problem, i.e., a roommate problem in which there is no stable matching.

${ }^{4}$ Irving (1985) presents an algorithm that either outputs a stable matching or "no" if none exists. Using Irving's (1985) algorithm, Tan (1991, Theorem 6.7) provides a necessary and sufficient condition for the existence of a stable matching in roommate problems.

${ }^{5}$ The maximum and the average are taken over all pairs of matched agents.
} 
rank gap in terms of the maximal disagreement, where the latter maximum is taken over all agents. Moreover, we show that the bound is "tight" (Proposition 1): for any $n$, there is a solvable roommate problem with $n$ agents such that there is a stable matching whose maximal rank gap coincides with the bound given in Theorem 1. Next, we focus on the average rank gap. Obviously, our upper bound of the maximal rank gap is also an upper bound of the average rank gap. Theorem 2 provides another upper bound of the average rank gap in terms of the average disagreement, where the latter average is taken over all agents. By means of two examples we show that neither bound is always better than the other (Examples 1 and 2).

Section 4 delivers (ii). We first clarify what we mean by the "size" of the core. From the point of view of a social planner or a clearinghouse, the number of stable matchings could be a relevant measure. However, we believe that from the point of view of an individual agent a more relevant measure is the range of the ranks of mates at stable matchings. Thus, we measure the size of the core by means of the rank gap that exists between each agent's worst and best stable mates, i.e., his least preferred and most preferred partners among those that are obtained at stable matchings. Similarly to Section 3, there are two natural ways to proceed: first, we consider the maximal rank gap in the core, and second, we consider the average rank gap in the core; the maximum and the average are taken over all agents. ${ }^{6} \mathrm{We}$ show that a (trivial) upper bound for the maximal rank gap in the core is tight (Proposition 2). Regarding the average rank gap in the core, we provide an upper bound in terms of average disagreement (Theorem 3) and show that it is "essentially" tight (Proposition 3).

Our analysis is closely related to Holzman and Samet (2014) which studies stable matchings between men and women in (two-sided) marriage problems (Gale and Shapley, 1962). Their results show that if for each side of the market rankings are sufficiently similar, then stable matchings are "assortative" and the size of the core is small. As Holzman and Samet (2014) point out, their results suggest similarity of rankings as a possible explanation of two real-life phenomena: matching of likes and smallness of the core. To study roommate problems we adapt the tools and the approach of Holzman and Samet (2014). Since our analysis in Sections 3 and 4 parallels the analysis of Holzman and Samet (2014) it is convenient to compare the results and proofs in detail in a separate section (Section 5).

Even though our analysis is similar to Holzman and Samet's (2014), it is non-trivial and of interest for the following reasons. First, roommate problems are particular instances of hedonic coalition formation (Bogomolnaia and Jackson, 2002) as well as network formation (Jackson and Watts, 2002) ${ }^{7}$ and appear in many real-life situations, e.g., assignment of students to double rooms in dorms, pairing police officers on patrols, exchange of holiday homes, etc. Second, assortativeness acquires a particular meaning in the case of roommate

\footnotetext{
${ }^{6}$ Note the difference between "average/maximal rank gap (between mates)" (studied in Section 3) and "average/maximal rank gap in the core" (studied in Section 4).

${ }^{7}$ We refer to Demange and Wooders (2004) and Jackson (2008) for surveys on coalition and network formation.
} 
problems. More specifically, in roommate problems all agents are peers (i.e., of the same type). Hence, it is natural to see if, conditional on stability, it is possible to achieve "rankfairness within matched pairs," i.e., the two members of each matched pair rank each other "similarly." Third, roommate problems are related to marriage problems, but they are structurally different. 8 The one-sidedness of roommate problems introduces challenging technical complications, in particular to establish the tightness of our bounds. We postpone further discussion to Section 5. Fourth, even though the literature on roommate problems has grown substantially, ${ }^{9}$ the structure of their core does not seem to be much explored.

The use of disagreements, rank gaps, and the size of the core in our analysis as well as that of Holzman and Samet (2014) requires that these measures have a similar meaning for different agents. This seems reasonable for "non-extreme" situations where variations of preference intensities are limited. But if for instance agents can be unacceptable (i.e., an agent prefers to remain single), preference intensities need no longer be limited. For this reason we assume throughout that all agents are mutually acceptable. We discuss this issue in more detail in Section 5.

Apart from Holzman and Samet (2014), our paper is also related to Pittel (1993). He studies roommate problems with preferences that are randomly and independently generated from the uniform distribution (where all agents are mutually acceptable). The most closely related part of his paper focuses on the ranks of stable mates, i.e., not rank gaps between stable mates (as in our study). For any stable matching $\mu$, let $\rho^{M}(\mu)$ denote the maximal rank of some agent's mate at $\mu$ (here the maximum is taken over all agents) and let $\rho^{A}(\mu)$ denote the average rank of the agents' mates at $\mu$ (here the average is taken over all agents). Pittel (1993, Theorem 3) shows that $\rho^{M}(\mu)$ is asymptotic, in probability, to $\sqrt{n} \ln (n)$, uniformly over all stable matchings $\mu$. Pittel (1993, Theorem 4) implies that $\rho^{A}(\mu)$ is asymptotic to $\sqrt{n}$, uniformly over all stable matchings $\mu$, with exponentially high probability. Therefore, when stable matchings exist, they are likely to be "well-balanced," in the sense that at each stable matching, matched agents "are likely to be close" to the top of each other's preference lists.

The remainder of the paper is organized as follows. In Section 2, we describe the roommate problem. In Sections 3 and 4, we present our results on the rank-fairness of stable matchings and the size of the core, respectively. In Section 5, we compare our results and proofs with those in Holzman and Samet (2014). Section 5 also discusses assumptions and possible extensions.

\footnotetext{
${ }^{8}$ For instance, unlike roommate problems, the core of any marriage problem is non-empty (Gale and Shapley, 1962, Theorem 1) and is a distributive lattice (Roth and Sotomayor, 1990, Theorems 2.16 and 3.8).

${ }^{9}$ We refer to the books of Gusfield and Irving (1989) and Manlove (2013) and the review of Gudmundsson (2014) for comprehensive overviews of the literature on roommate problems.
} 


\section{Model}

There is a finite set of agents $N=\{1,2, \ldots, n\}$ where $n \geq 2$ is a positive integer. Each agent $i$ has a strict preference relation over being matched to another agent in $N \backslash\{i\}$ and being unmatched (or having an outside option) which is denoted by $i$. For each $i$, agent $i$ 's preferences can be represented by a ranking, i.e., a bijection $r_{i}: N \rightarrow N$ such that for $j, j^{\prime} \in N, r_{i}(j)<r_{i}\left(j^{\prime}\right)$ if and only if agent $i$ prefers $j$ to $j^{\prime}$. The integer $r_{i}(j)$ is the rank of $j$ in agent $i$ 's ranking. Hence, more preferred agents have a smaller rank. In particular, the agent ranked first is $i$ 's most preferred roommate, the agent ranked second is $i$ 's second most preferred agent, and so on. We adopt the quite common assumption from the literature that being unmatched is each agent's least preferred option, i.e., for each $i \in N, r_{i}(i)=n$. Let $r \equiv\left(r_{i}\right)_{i \in N}$ be the profile of rankings. A (roommate) problem (Gale and Shapley, 1962) is given by $(N, r)$, or shortly $r$.

A matching is a function $\mu: N \rightarrow N$ of order two, i.e., for all $i \in N, \mu(\mu(i))=i$. If $j=\mu(i)$ then we say that $i$ and $j$ are matched to one another and that they are (each other's) mates at $\mu$. Equivalently, a matching can be written as a partition of $N$ in pairs and singletons.

A pair $\{i, j\} \subseteq N$ is a blocking pair for matching $\mu$ if $r_{i}(j)<r_{i}(\mu(i))$ and $r_{j}(i)<r_{j}(\mu(j))$. A matching $\mu$ is stable if there is no blocking pair. Let $\boldsymbol{S}(\boldsymbol{r})$ denote the set of stable matchings at $r$. A coalition $T \subseteq N$ is a blocking coalition for matching $\mu$ if there exists a matching $\mu^{\prime}$ such that $\mu^{\prime}(T)=T$, for all $i \in T, r_{i}\left(\mu^{\prime}(i)\right) \leq r_{i}(\mu(i))$ and for some $j \in T, r_{j}\left(\mu^{\prime}(j)\right)<r_{j}(\mu(j))$. The core is the set of matchings that cannot be blocked by any coalition. Alcalde (1994) shows that the core equals $S(r)$. A roommate problem is solvable if its core is non-empty. Not all roommate problems are solvable (Gale and Shapley, 1962, Example 3). We focus on the class of solvable roommate problems and study their stable matchings. The following observation follows from the "lone wolf theorem" (Klaus and Klijn, 2010, Theorem 1) and the assumption that all agents are mutually acceptable.

Observation 1. Let $r$ be solvable. If $n$ is even, then each stable matching consists of exactly $\frac{n}{2}$ pairs of agents. If $n$ is odd, then there is an agent ("the lone wolf") that is the unique unmatched agent at all stable matchings.

In the case of a solvable roommate problem with an odd number of agents, we will assume, without loss of generality, that the lone wolf of Observation 1 is agent $n$.

\section{Rank-fairness of stable matchings}

Let $r$ be a solvable roommate problem and $\mu \in S(r)$. Matching $\mu$ is rank-fair (within matched pairs) if no agent is treated more favorably than his mate at $\mu$ in the sense that they have 
the same rank in each other's ranking. Formally, matching $\mu$ is rank-fair (within matched pairs) if for all $i \in N, r_{i}(\mu(i))=r_{\mu(i)}(i)$.

Observation 2. If all agents' rankings are based on a (common) ordering of the agents, then there is a unique stable matching that is, moreover, rank-fair. Formally and without loss of generality, for each $i \in N$, let $r_{i}: 1,2, \ldots, i-1, i+1, \ldots, n-1, n$ denote (from most to least preferred) agent $i$ 's ranking. Then, $S(r)$ contains a unique stable matching that is, moreover, rank-fair.

Our objective is to show that Observation 2 is "robust" in multiple ways.

Let $r$ be a solvable roommate problem. The (dis)similarity of rankings is measured through "disagreements." For each $i \in N$, the disagreement that the agents in $N\{i\}$ have over agent $i$ is given by $\boldsymbol{\delta}^{\boldsymbol{r}}(\boldsymbol{i}) \equiv \max _{j \in N \backslash\{i\}} r_{j}(i)-\min _{j \in N \backslash\{i\}} r_{j}(i)$. The maximal disagreement at $\boldsymbol{r}$ is given by

$$
\boldsymbol{\Delta}^{M}(\boldsymbol{r}) \equiv \max _{i \in N} \delta^{r}(i)
$$

Let $\mu \in S(r)$. Rank-(un)fairness of $\mu$ is measured through the rank gaps (or $\boldsymbol{r}$-gaps) $\boldsymbol{\gamma}^{\boldsymbol{r}}(\boldsymbol{i}, \boldsymbol{j}) \equiv\left|r_{i}(j)-r_{j}(i)\right|$ where $i$ and $j$ are mates at $\mu$. Large rank gaps indicate that some agents are treated unfairly (asymmetrically) with respect to their mates. The maximal $\boldsymbol{r}$-gap between mates at $\boldsymbol{\mu}$ is given by

$$
\Gamma^{M}(\boldsymbol{r}, \boldsymbol{\mu}) \equiv \max _{\{i, j\} \in \mu} \gamma^{r}(i, j)
$$

Our first result, Theorem 1, provides an upper bound for the maximal rank gap between mates at stable matchings in terms of maximal disagreement. This shows the robustness of Observation 2 with respect to rank-fairness: if rankings are sufficiently similar $\left(\Delta^{M}(r)\right.$ is small), then no stable matching can be very rank-unfair (for each $\mu \in S(r), \Gamma^{M}(r, \mu)$ is small).

Theorem 1. [Bound for maximal rank gap between mates.]

Let $r$ be a solvable roommate problem. Then, for each stable matching $\mu \in S(r)$,

$$
\Gamma^{M}(r, \mu) \leq \boldsymbol{B}^{\mathbf{1}}(\boldsymbol{r}) \equiv \begin{cases}0 & \text { if } n=2,3 \\ 2 & \text { if } n=4,5 \\ 2 \Delta^{M}(r)-1 & \text { if } n \geq 6\end{cases}
$$

Proof. Let $r$ be a solvable roommate problem. Let $n=2$. Then, $r_{i}(j)=1$ for all $i, j \in N$ with $i \neq j$ and at the unique stable matching $\mu$ the two agents are matched to one another. One immediately verifies that $\Gamma^{M}(r, \mu)=0$.

Let $n=3$. Then, since agent 3 is the lone wolf, agents 1 and 2 are matched to one another at the unique stable matching, and moreover $r_{1}(2)=r_{2}(1)=1$. One immediately verifies that $\Gamma^{M}(r, \mu)=0$.

Let $n=4$. Then, for each stable matching $\mu$ and each $\{i, j\} \in \mu, \gamma^{r}(i, j) \leq 3-1=2$. Hence, $\Gamma^{M}(r, \mu) \leq 2$. 
Let $n=5$. Then, for each stable matching $\mu$ and each $\{i, j\} \in \mu, \gamma^{r}(i, j) \leq 3-1=2$. (If for some agent $i \neq n, r_{i}(\mu(i))=4$, then $\{i, 5\}$ would constitute a blocking pair for $\mu$.) Hence, $\Gamma^{M}(r, \mu) \leq 2$.

Let $n \geq 6$. Let $\mu \in S(r)$ and $\{i, j\}$ be a pair of mates at $\mu$, i.e., $\mu(i)=j$ and $i \neq j$. We prove that $r_{i}(j)-r_{j}(i) \leq 2 \Delta^{M}(r)-1$.

By definition of $r_{i}(j)$, agent $i$ prefers each of the $r_{i}(j)-1$ agents in $J^{\prime} \equiv\left\{j^{\prime} \in N: r_{i}\left(j^{\prime}\right)<\right.$ $\left.r_{i}(j)\right\}$ to agent $j$. Let $j^{\prime} \in J^{\prime}$. Since $\mu$ is stable, $r_{j^{\prime}}\left(i^{\prime}\right)<r_{j^{\prime}}(i)$ where $i^{\prime} \equiv \mu\left(j^{\prime}\right)$. Then, $0<-r_{j^{\prime}}\left(i^{\prime}\right)+r_{j^{\prime}}(i)$, which is equivalent to $\left[r_{j}\left(i^{\prime}\right)-r_{j}(i)\right]<\left[r_{j}\left(i^{\prime}\right)-r_{j}(i)\right]-r_{j^{\prime}}\left(i^{\prime}\right)+r_{j^{\prime}}(i)$. Since $r_{j}\left(i^{\prime}\right)-r_{j^{\prime}}\left(i^{\prime}\right) \leq \delta^{r}\left(i^{\prime}\right) \leq \Delta^{M}(r)$ and $r_{j^{\prime}}(i)-r_{j}(i) \leq \delta^{r}(i) \leq \Delta^{M}(r)$, we have $r_{j}\left(i^{\prime}\right)-r_{j}(i)<$ $2 \Delta^{M}(r)$. Hence, $r_{j}\left(i^{\prime}\right)<r_{j}(i)+2 \Delta^{M}(r)$. Therefore, $\mu\left(j^{\prime}\right)$ is among the $r_{j}(i)+2 \Delta^{M}(r)-1$ most preferred options of agent $j$. Hence, since $\left|J^{\prime}\right|=r_{i}(j)-1$ and for all $j^{\prime \prime}, j^{\prime \prime \prime} \in J^{\prime}$ with $j^{\prime \prime} \neq j^{\prime \prime \prime}$, $\mu\left(j^{\prime \prime}\right) \neq \mu\left(j^{\prime \prime \prime}\right)$, we have that all $r_{i}(j)-1$ agents in $\mu\left(J^{\prime}\right)$ are among the $r_{j}(i)+2 \Delta^{M}(r)-1$ most preferred options of agent $j$.

Now notice that trivially $\Delta^{M}(r)>0$ and hence $r_{j}(i)<r_{j}(i)+2 \Delta^{M}(r)$. So, $i$ is also among the $r_{j}(i)+2 \Delta^{M}(r)-1$ most preferred options of agent $j$. Since $i=\mu(j)$ and $j \notin J^{\prime}$, $i \notin \mu\left(J^{\prime}\right)$. Hence, there are $\left|\mu\left(J^{\prime}\right) \cup\{i\}\right|=r_{i}(j)-1+1$ agents among the $r_{j}(i)+2 \Delta^{M}(r)-1$ most preferred options of agent $j$. Hence, $r_{i}(j) \leq r_{j}(i)+2 \Delta^{M}(r)-1$, which completes the proof.

Next, we show that for each $n \geq 2$ the bound provided in Theorem 1 is in fact "tight."

Proposition 1. [Tightness of bound for maximal rank gap between mates.]

For each $n \geq 2$, there is a solvable roommate problem $r$ such that for some stable matching $\mu \in S(r)$,

$$
\Gamma^{M}(r, \mu)=B^{1}(r)
$$

Proof. The cases $n=2,3$ follow from the proof of Theorem 1 .

Let $n=4$. Consider the problem $(N, r)$ with $N=\{1,2,3,4\}$ and $r$ given on the left hand side of Table 1. Each column represents the ranking of an agent where higher placed agents are more preferred agents. For instance, column 1 shows that agent 1's most preferred roommate is agent 2 , his second most preferred roommate is agent 3, and his third most preferred roommate is agent 4. Since being unmatched is each agent's least preferred option, we have omitted the option of being unmatched in the table. The unique stable matching at $r$ is $\mu=\{\{1,2\},\{3,4\}\}$, the boxed matching in Table 1. So, $r$ is solvable. Since $\gamma^{r}(1,2)=0$ and $\gamma^{r}(3,4)=2, \Gamma^{M}(r, \mu)=2$. Hence, $\Gamma^{M}(r, \mu)=2=B^{1}(r)$. The proof of the case $n=5$ runs similarly using the profile of rankings on the right hand side of Table 1.

Let $n=6$. Consider the problem $(N, r)$ with $N=\{1,2, \ldots, 6\}$ and $r$ given by the left hand side of Table 2. The unique stable matching at $r$ is $\mu=\{\{1,2\},\{3,4\},\{5,6\}\}$, the boxed matching in Table 2. So, $r$ is solvable. Since $\gamma^{r}(1,2)=3, \gamma^{r}(3,4)=0$, and $\gamma^{r}(5,6)=0$, 


\begin{tabular}{cccc}
\hline \hline$r_{1}$ & $r_{2}$ & $r_{3}$ & $r_{4}$ \\
\hline 2 & 1 & 1 & 3 \\
3 & 3 & 2 & 1 \\
4 & 4 & 4 & 2 \\
\hline \hline
\end{tabular}

\begin{tabular}{ccccc}
\hline \hline$r_{1}$ & $r_{2}$ & $r_{3}$ & $r_{4}$ & $r_{5}$ \\
\hline 2 & 1 & 1 & 3 & 3 \\
3 & 3 & 2 & 1 & 1 \\
4 & 4 & 4 & 2 & 2 \\
5 & 5 & 5 & 5 & 4 \\
\hline \hline
\end{tabular}

Table 1: Rankings in Proposition 1 for $n=4$ (left) and $n=5$ (right).

\begin{tabular}{cccccc}
\hline \hline$r_{1}$ & $r_{2}$ & $r_{3}$ & $r_{4}$ & $r_{5}$ & $r_{6}$ \\
\hline 3 & 3 & 4 & 3 & 3 & 3 \\
4 & 1 & 1 & 1 & 4 & 4 \\
5 & 4 & 5 & 5 & 6 & 5 \\
6 & 5 & 6 & 6 & 1 & 1 \\
2 & 6 & 2 & 2 & 2 & 2 \\
\hline \hline
\end{tabular}

\begin{tabular}{ccccccc}
\hline \hline$r_{1}$ & $r_{2}$ & $r_{3}$ & $r_{4}$ & $r_{5}$ & $r_{6}$ & $r_{7}$ \\
\hline 3 & 3 & 4 & 3 & 3 & 3 & 3 \\
4 & 1 & 1 & 1 & 4 & 4 & 1 \\
5 & 4 & 5 & 5 & 6 & 5 & 4 \\
6 & 5 & 6 & 6 & 1 & 1 & 5 \\
2 & 6 & 2 & 2 & 2 & 2 & 6 \\
7 & 7 & 7 & 7 & 7 & 7 & 2 \\
\hline \hline
\end{tabular}

Table 2: Rankings in Proposition 1 for $n=6$ (left) and $n=7$ (right).

we have $\Gamma^{M}(r, \mu)=3$. It is easy to verify that $\delta^{r}(1)=\delta^{r}(4)=\delta^{r}(6)=2, \delta^{r}(5)=1$, and $\delta^{r}(2)=\delta^{r}(3)=0$. Hence, $\Delta^{M}(r)=2$. So, $\Gamma^{M}(r, \mu)=3=2 \Delta^{M}(r)-1=B^{1}(r)$. The proof of the case $n=7$ runs similarly using the profile of rankings on the right hand side of Table 2 .

Let $n \geq 8$ be even. We construct a problem $(N, r)$ with $N=\{1,2, \ldots, n\}$ as follows. For convenience, Table 3 illustrates our construction for $n=10$. First, we require that the agents in $\{1,2, \ldots, 6\}$ order their options in the same way as in Table 2 (see boldfaced part of Table 3)- we will refer to this condition as "restricted problem." Second, we impose the following additional conditions on the rankings:

(1) For each agent $j \in\{7,8, \ldots, n\}, r_{2}(j)=j-1$.

(2) For each agent $i \in N \backslash\{2\}, r_{i}(2)=n-1$.

(3) For each agent $i \in\{1,3,4,5,6\}$ and each agent $j \in\{7,8, \ldots, n\}, r_{i}(j)=j-2$.

(4) Let $i \in\{8,10, \ldots, n\}$ be even. Then, (4a) for each agent $j \in\left\{3,4, \ldots, \frac{i}{2}+1\right\}, r_{i}(j)=j-2$, (4b) $r_{i}(i-1)=\frac{i}{2},(4 \mathrm{c}) r_{i}(1)=\frac{i}{2}+1$, and (4d) for each agent $j \in\left\{\frac{i}{2}+2, \frac{i}{2}+3, \ldots, i-2\right\}$, $r_{i}(j)=j$.

(5) For each even agent $i \in\{8,10, \ldots, n-2\}$ and each agent $j \in\{i+1, \ldots, n\}, r_{i}(j)=j-2$.

(6) For each odd agent $i \in\{7,9, \ldots, n-1\}$ and each agent $j \in N \backslash\{i, i+1\}$, (6a) $r_{i}(j)=$ $r_{i+1}(j)$ and $(6 \mathrm{~b}) r_{i}(i+1)=\frac{i+1}{2}$. 
(6a) (4a)

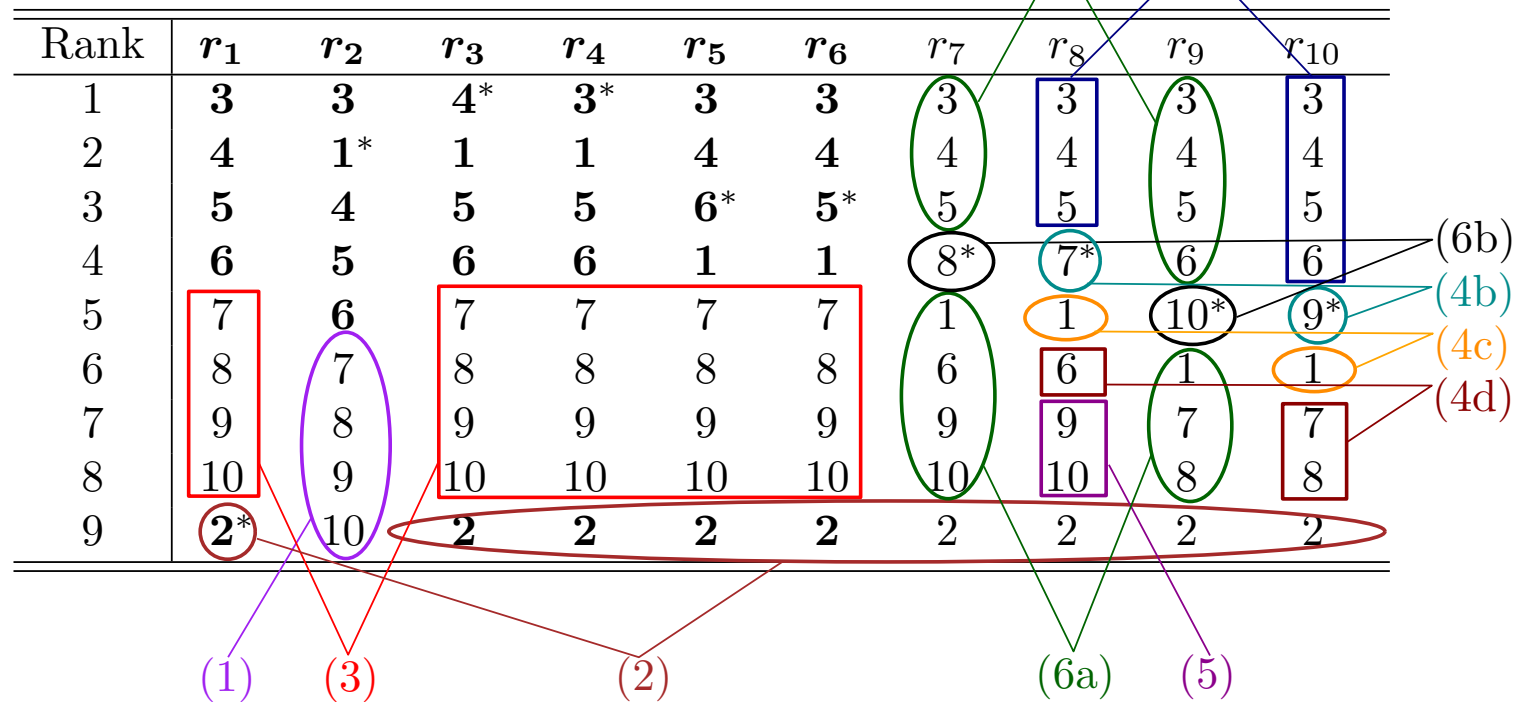

Table 3: Rankings in Proposition 1 for $n=10$ (the boldfaced part is "consistent" with Table 2).

Consider the matching $\mu$ such that for each odd agent $i \in N, \mu(i)=i+1$ (the starred matching in Table 3). We show that $\mu$ is stable at $r$. Let $\sigma$ be the order such that $\sigma=$ $\left(\sigma_{1}, \sigma_{2}, \ldots, \sigma_{n}\right)=(3,4,5,6, \ldots, n-1, n, 1,2)$. For each odd $k \in\{1,3, \ldots, n-1\},\left\{\sigma_{k}, \sigma_{k+1}\right\} \in \mu$. By the restricted problem, (4a), (4b), and (6), for each odd $k \in\{1,3, \ldots, n-1\}$ and each $l>k+1$, we have $r_{\sigma_{k}}\left(\sigma_{k+1}\right)<r_{\sigma_{k}}\left(\sigma_{l}\right)$ and $r_{\sigma_{k+1}}\left(\sigma_{k}\right)<r_{\sigma_{k}}\left(\sigma_{l}\right)$. Hence, for each odd $k \in$ $\{1,3, \ldots, n-1\}, \sigma_{k}$ and $\sigma_{k+1}$ are each other's most preferred agent in $N \backslash\left\{\sigma_{1}, \sigma_{2}, \ldots, \sigma_{k-1}\right\}$. Hence, $\mu$ is stable. (So, in particular, $r$ is solvable.) For each odd $k \in\{3,5, \ldots, n-1\}$, $\gamma^{r}\left(\sigma_{k}, \sigma_{k+1}\right)=0$ and $\gamma^{r}\left(\sigma_{1}, \sigma_{2}\right)=r_{1}(2)-r_{2}(1)=(n-1)-2=n-3$. Hence, $\Gamma^{M}(r, \mu)=n-3$.

Next, we calculate for each $i \in N$, the disagreement over agent $i, \delta^{r}(i)$, to determine $\Delta^{M}(r)=\max _{i \in N} \delta^{r}(i)$.

(i) By the restricted problem, (4c), and (6a), $\delta^{r}(1)=r_{n}(1)-r_{2}(1)=\left(\frac{n}{2}+1\right)-2=\frac{n}{2}-1$.

(ii) $\operatorname{By}(2), \delta^{r}(2)=0$.

(iii) By the restricted problem, (4a), and (6a), $\delta^{r}(3)=0, \delta^{r}(4)=2$, and $\delta^{r}(5)=1$.

(iv) By (4) and (6), for each odd agent $i \in\{7,9, \ldots, n-3\}, \delta^{r}(i)=r_{i+2}(i)-r_{i+1}(i)=i-\left(\frac{i+1}{2}\right)$. The maximum of $\delta^{r}(i)$ is achieved at $i=n-3$ where $\delta^{r}(n-3)=r_{n-1}(n-3)-r_{n-2}(n-$ $3)=(n-3)-\left(\frac{n-3+1}{2}\right)=\frac{n}{2}-2$.

(v) By the restricted problem, (4), and (6), for each even agent $i \in\{6,8, \ldots, n-2\}, \delta^{r}(i)=$ $r_{i+1}(i)-r_{i-1}(i)=i-\frac{i}{2}$. The maximum of $\delta^{r}(i)$ is achieved at $i=n-2$ where $\delta^{r}(n-2)=r_{n-1}(n-2)-r_{n-3}(n-2)=(n-2)-\left(\frac{n-2}{2}\right)=\frac{n}{2}-1$. 
(vi) By (1), (3), (4b), (5), and (6a), $\delta^{r}(n-1)=r_{2}(n-1)-r_{n}(n-1)=(n-2)-\left(\frac{n}{2}\right)=\frac{n}{2}-2$.

(vii) By (1), (3), (5), and (6), $\delta^{r}(n)=r_{2}(n)-r_{n-1}(n-2)=(n-1)-\left(\frac{n}{2}\right)=\frac{n}{2}-1$.

Finally, from (i)-(vii) it follows that $\Delta^{M}(r)=\max _{i \in N} \delta^{r}(i)=\frac{n}{2}-1$. Hence, we have $B^{1}(r)=2 \Delta^{M}(r)-1=2\left(\frac{n}{2}-1\right)-1=n-3=\Gamma^{M}(r, \mu)$.

Let $n \geq 9$ be odd. Consider the problem constructed for the case of $n-1$ agents. We add agent $n$ and define the ranks $r_{i}(n)=n-1$ for all $i=1,2, \ldots, n-1$ and the ranking $r_{n}$ such that (a) for all $j=1,3,4, \ldots, n-1, r_{n}(j)=r_{2}(j)$ and (b) $r_{n}(2)=n-1$. The same arguments as for the case $n-1$ show that $B^{1}(r)=2 \Delta^{M}(r)-1=2\left(\left\lfloor\frac{n}{2}\right\rfloor-1\right)-1=2\left(\frac{n-1}{2}-1\right)-1=$ $n-4=\Gamma^{M}(r, \mu)$. (The only difference is that $\delta^{r}(2)=1$ and $\delta^{r}(n)=0$ but this does not affect the computation of $\Delta^{M}(r)$.)

An alternative approach to quantify the rank-(un)fairness of a stable matching is to look at the average rank gap instead of the maximal rank gap. Let $r$ be a solvable roommate problem and $\mu \in S(r)$. The average $\boldsymbol{r}$-gap between mates at $\boldsymbol{\mu}$ is defined as ${ }^{10}$

$$
\Gamma^{\boldsymbol{A}}(\boldsymbol{r}, \boldsymbol{\mu}) \equiv \frac{1}{\lfloor n / 2\rfloor} \sum_{\{i, j\} \in \mu} \gamma^{r}(i, j) .
$$

An immediate consequence of Theorem 1 is that $\Gamma^{A}(r, \mu) \leq B^{1}(r)$.

To study average rank gaps between mates at stable matchings, we can also look at the average disagreement instead of the maximal disagreement. The average disagreement at $\boldsymbol{r}$ is defined as

$$
\boldsymbol{\Delta}^{\boldsymbol{A}}(\boldsymbol{r}) \equiv \frac{1}{n} \sum_{i \in N} \delta^{r}(i)
$$

The next result, Theorem 2, shows that the average rank gap between mates at a stable matching can be bounded by means of the average disagreement. This shows again the robustness of Observation 2 with respect to rank-fairness, albeit in a slightly different way: if rankings are sufficiently similar $\left(\Delta^{A}(r)\right.$ is small), then no stable matching can be very rank-unfair (for each $\mu \in S(r), \Gamma^{A}(r, \mu)$ is small).

Theorem 2. [Bound for average rank gap between mates.]

Let $r$ be a solvable roommate problem. Then, for each stable matching $\mu \in S(r)$,

$$
\Gamma^{A}(r, \mu) \leq \boldsymbol{B}^{\mathbf{2}}(\boldsymbol{r}) \equiv \begin{cases}4 \Delta^{A}(r) & \text { if } n \text { is even } \\ 4 \frac{n}{n-1} \Delta^{A}(r) & \text { if } n \text { is odd }\end{cases}
$$

Proof. Let $r$ be a solvable problem. Let $\mu \in S(r)$. Let $N^{0} \equiv\left\{i \in N: r_{i}(\mu(i))>r_{\mu(i)}(i)\right\}$. Note that $\left|N^{0}\right| \leq\left\lfloor\frac{n}{2}\right\rfloor$. If $N^{0}=\emptyset$, then the statement follows trivially.

\footnotetext{
${ }^{10}$ For any real number $x,\lfloor x\rfloor$ is the largest integer $k$ with $k \leq x$. Recall that any stable matching contains exactly $\left\lfloor\frac{n}{2}\right\rfloor$ pairs of agents (and additionally one "lone wolf" in the case of an odd number of agents).
} 
Suppose $N^{0} \neq \emptyset$. Let $i^{0} \in N^{0}$. Let $M_{i^{0}}$ be the set of $r_{i^{0}}\left(\mu\left(i^{0}\right)\right)-1$ agents $\mu(j)$ with $j \in N$ such that $i^{0}$ strictly prefers agent $\mu(j)$ to $\mu\left(i^{0}\right)$. Since $\mu \in S(r)$, for each $j \in N$ with $\mu(j) \in M_{i^{0}}$ we have

$$
r_{\mu(j)}(j)<r_{\mu(j)}\left(i^{0}\right) .
$$

Next, note that there are $\max _{i \in N \backslash\left\{i^{0}\right\}} r_{i}\left(i^{0}\right)-1$ agents in $N$ that in $r_{i^{0}}$ obtain a rank that is strictly smaller than $\max _{i \in N \backslash\left\{i^{0}\right\}} r_{i}\left(i^{0}\right)$. Hence, there are at least

$$
\max \left\{0,\left(r_{i^{0}}\left(\mu\left(i^{0}\right)\right)-1\right)-\left(\max _{i \in N \backslash\left\{i^{0}\right\}} r_{i}\left(i^{0}\right)-1\right)\right\}=\max \left\{0, r_{i^{0}}\left(\mu\left(i^{0}\right)\right)-\max _{i \in N \backslash\left\{i^{0}\right\}} r_{i}\left(i^{0}\right)\right\}
$$

agents $j \in N$ with $\mu(j) \in M_{i^{0}}$ that in $r_{i^{0}}$ obtain a rank that is weakly larger than the number $\max _{i \in N \backslash\left\{i^{0}\right\}} r_{i}\left(i^{0}\right)$, i.e., $r_{i^{0}}(j) \geq \max _{i \in N \backslash\left\{i^{0}\right\}} r_{i}\left(i^{0}\right)$. Note that $r_{i^{0}}(j) \geq \max _{i \in N \backslash\left\{i^{0}\right\}} r_{i}\left(i^{0}\right)$ implies $\max _{i \in N \backslash\{j\}} r_{i}(j) \geq \max _{i \in N \backslash\left\{i^{0}\right\}} r_{i}\left(i^{0}\right)$ (here we use that $j \neq i^{0}$, which follows from $\mu(j) \in M_{i^{0}}$ ). Hence, there are at least $\max \left\{0, r_{i^{0}}\left(\mu\left(i^{0}\right)\right)-\max _{i \in N \backslash\left\{i^{0}\right\}} r_{i}\left(i^{0}\right)\right\}$ agents $j \in N$ with $\mu(j) \in$ $M_{i^{0}}$ and that satisfy

$$
\max _{i \in N \backslash\{j\}} r_{i}(j) \geq \max _{i \in N \backslash\left\{i^{0}\right\}} r_{i}\left(i^{0}\right) .
$$

Let $P_{i^{0}}$ be the set of agents $j$ with $\mu(j) \in M_{i^{0}}$ that satisfy (1) and (2). From the above it immediately follows that

$$
\left|P_{i^{0}}\right| \geq r_{i^{0}}\left(\mu\left(i^{0}\right)\right)-\max _{i \in N \backslash\left\{i^{0}\right\}} r_{i}\left(i^{0}\right) .
$$

Let $P \equiv \cup_{i^{0} \in N^{0}} P_{i^{0}}$. For each $j \in P$, let $Q_{j} \equiv\left\{i^{0} \in N^{0}: j \in P_{i^{0}}\right\}$. Since each $j \in P$ satisfies (1) for each $i^{0} \in Q_{j}$, agent $\mu(j)$ gives a smaller rank to $j$ than to each of the agents $i^{0}$ in $Q_{j}$, which implies that $r_{\mu(j)}(j)+\left|Q_{j}\right| \leq \max _{i^{0} \in Q_{j}} r_{\mu(j)}\left(i^{0}\right)$. Hence, for each $j \in P$,

$$
r_{\mu(j)}(j) \leq \max _{i^{0} \in Q_{j}} r_{\mu(j)}\left(i^{0}\right)-\left|Q_{j}\right| \leq \max _{i^{0} \in Q_{j}} \max _{i \in N \backslash\left\{i^{0}\right\}} r_{i}\left(i^{0}\right)-\left|Q_{j}\right|,
$$

where the second inequality follows from $\mu(j) \neq i^{0}$. (By definition of $M_{i^{0}}, i^{0}$ strictly prefers agent $\mu(j)$ to $\mu\left(i^{0}\right)$. Since $i^{0}$ is the worst option for $i^{0}$, it follows that $\mu(j) \neq i^{0}$.) Since each $j \in P$ satisfies (2) with respect to each $i^{0} \in Q_{j}$, we also have that for all $j \in P$,

$$
\max _{i \in N \backslash\{j\}} r_{i}(j) \geq \max _{i^{0} \in Q_{j}} \max _{i \in N \backslash\left\{i^{0}\right\}} r_{i}\left(i^{0}\right) .
$$

Inequalities (4) and (5) imply that for all $j \in P$,

$$
\left|Q_{j}\right| \leq \max _{i \in N \backslash\{j\}} r_{i}(j)-r_{\mu(j)}(j) \leq \max _{i \in N \backslash\{j\}} r_{i}(j)-\min _{i \in N \backslash\{j\}} r_{i}(j)=\delta^{r}(j),
$$

where the second inequality follows from $j \neq \mu(j)$ (because $\mu$ is stable). Then,

$$
\sum_{i^{0} \in N^{0}}\left[r_{i^{0}}\left(\mu\left(i^{0}\right)\right)-\max _{i \in N \backslash\left\{i^{0}\right\}} r_{i}\left(i^{0}\right)\right] \leq \sum_{i^{0} \in N^{0}}\left|P_{i^{0}}\right|=\sum_{j \in P}\left|Q_{j}\right| \leq \sum_{j \in P} \delta^{r}(j) \leq \sum_{i \in N} \delta^{r}(i),
$$


where the first and second inequalities follow from (3) and (6), respectively. Moreover,

$$
\sum_{i^{0} \in N^{0}}\left[\max _{i \in N \backslash\left\{i^{0}\right\}} r_{i}\left(i^{0}\right)-r_{\mu\left(i^{0}\right)}\left(i^{0}\right)\right] \leq \sum_{i^{0} \in N^{0}}\left[\max _{i \in N \backslash\left\{i^{0}\right\}} r_{i}\left(i^{0}\right)-\min _{i \in N \backslash\left\{i^{0}\right\}} r_{i}\left(i^{0}\right)\right]=\sum_{i^{0} \in N^{0}} \delta^{r}\left(i^{0}\right) .
$$

Adding inequalities (7) and (8) yields

$$
\sum_{i^{0} \in N^{0}}\left[r_{i^{0}}\left(\mu\left(i^{0}\right)\right)-r_{\mu\left(i^{0}\right)}\left(i^{0}\right)\right] \leq \sum_{i \in N} \delta^{r}(i)+\sum_{i^{0} \in N^{0}} \delta^{r}\left(i^{0}\right) \leq 2 \sum_{i \in N} \delta^{r}(i)
$$

Hence,

$$
\begin{aligned}
\Gamma^{A}(r, \mu) & =\frac{1}{\lfloor n / 2\rfloor} \sum_{\{i, j\} \in \mu}\left|r_{i}(j)-r_{j}(i)\right|=\frac{1}{\lfloor n / 2\rfloor} \sum_{i^{0} \in N^{0}}\left[r_{i^{0}}\left(\mu\left(i^{0}\right)\right)-r_{\mu\left(i^{0}\right)}\left(i^{0}\right)\right] \\
& \leq \frac{2}{\lfloor n / 2\rfloor} \sum_{i \in N} \delta^{r}(i)=\frac{2 n}{\lfloor n / 2\rfloor} \Delta^{A}(r)=B^{2}(r) .
\end{aligned}
$$

We have established that $B^{1}$ and $B^{2}$ are both bounds for $\Gamma^{A}(r, \mu)$, but how do they compare? Bound $B^{1}$ gives a sharper estimate for roommate problems where the maximal disagreement is "small" relative to the average disagreement. Bound $B^{2}$, on the other hand, is more useful for roommate problems where the maximal disagreement is "large" relative to the average disagreement. We illustrate this in the following two examples where we exhibit solvable roommate problems $r^{A}$ and $r^{B}$ such that $B^{1}\left(r^{A}\right)>B^{2}\left(r^{A}\right)$ and $B^{2}\left(r^{B}\right)>B^{1}\left(r^{B}\right)$.

Example 1. [Solvable roommate problem $r^{A}$ with $B^{1}\left(r^{A}\right)>B^{2}\left(r^{A}\right)$.]

Consider the problem $\left(N, r^{A}\right)$ with $N=\{1,2, \ldots, 6\}$ and $r^{A}=r$ given by Table 4 . The unique stable matching at $r^{A}$ is $\mu=\{\{1,2\},\{3,4\},\{5,6\}\}$, the boxed matching in Table 4 . So, $r^{A}$ is solvable.

\begin{tabular}{cccccc}
\hline \hline$r_{1}$ & $r_{2}$ & $r_{3}$ & $r_{4}$ & $r_{5}$ & $r_{6}$ \\
\hline 3 & 3 & 4 & 3 & 3 & 3 \\
4 & 4 & 1 & 1 & 4 & 4 \\
5 & 5 & 5 & 5 & 6 & 5 \\
6 & 6 & 6 & 6 & 1 & 1 \\
2 & 1 & 2 & 2 & 2 & 2 \\
\hline \hline
\end{tabular}

Table 4: Rankings in Example 1.

It is easy to verify that $\delta^{r^{A}}(1)=3, \delta^{r^{A}}(2)=\delta^{r^{A}}(3)=\delta^{r^{A}}(5)=0$, and $\delta^{r^{A}}(4)=\delta^{r^{A}}(6)=1$. Hence, $B^{1}\left(r^{A}\right)=2 \Delta^{M}\left(r^{A}\right)-1=2 \times 3-1=5$ and $B^{2}\left(r^{A}\right)=4 \Delta^{A}\left(r^{A}\right)=4 \times \frac{3+0+0+1+0+1}{6}=\frac{10}{3}$. Therefore, $B^{1}\left(r^{A}\right)>B^{2}\left(r^{A}\right)$. 
Example 2. [Solvable roommate problem $r^{B}$ with $B^{2}\left(r^{B}\right)>B^{1}\left(r^{B}\right)$.]

Consider the problem $\left(N, r^{B}\right)$ with $N=\{1,2, \ldots, 6\}$ and $r^{B}=r$ given by the left hand side of Table 2. Note that the only (small) difference between $r^{B}$ and $r^{A}$ of Example 1 is the ranking of agent 2 . The unique stable matching at $r^{B}$ is $\mu=\{\{1,2\},\{3,4\},\{5,6\}\}$, the boxed matching in the left hand side of Table 2. So, $r^{B}$ is solvable.

It is easy to verify that $\delta^{r^{B}}(1)=\delta^{r^{B}}(4)=\delta^{r^{B}}(6)=2, \delta^{r^{B}}(2)=\delta^{r^{B}}(3)=0$, and $\delta^{r^{B}}(5)=1$. Hence, $B^{1}\left(r^{B}\right)=2 \Delta^{M}\left(r^{B}\right)-1=2 \times 2-1=3$ and $B^{2}\left(r^{B}\right)=4 \Delta^{A}\left(r^{B}\right)=4 \times \frac{2+0+0+2+1+2}{6}=\frac{14}{3}$. Therefore, $B^{2}\left(r^{B}\right)>B^{1}\left(r^{B}\right)$.

Examples 1 and 2 show that $B^{1}$ and $B^{2}$ are very conservative bounds for $\Gamma^{A}(r, \mu)$. It is easy to verify that for $n=4$, for each solvable roommate problem $r$, and for each stable matching $\mu \in S(r), \Gamma^{A}(r, \mu)<B^{1}(r)$. Similarly, for each $n \in\{3,4\}$, for each solvable roommate problem $r$, and for each stable matching $\mu \in S(r), \Gamma^{A}(r, \mu)<B^{2}(r)$. ${ }^{11}$ We have carried out computer simulations to study the performance of $B^{1}$ and $B^{2}$ for larger values of $n$. Our simulations suggest that strict inequalities hold for all $n>4$ as well, but we have not been able to formally prove this.

\section{Size of the core}

In this section, we show that Observation 2 is also robust in terms of the "size" of the core. Clearly, there are different ways to decide whether the core is "small" or "large." From the point of view of a social planner or a clearinghouse, the number of stable matchings could be a relevant measure. However, we believe that from the point of view of an individual agent a more relevant measure is the range of the ranks of mates at stable matchings. Thus, we measure the size of the core by means of the rank gap that exists between each agent's least preferred and most preferred partners among those that are obtained at stable matchings.

Formally, let $r$ be a solvable roommate problem. For each $i \in N$, a stable mate of $i$ is an agent that is matched to $i$ at some stable matching, i.e., $j$ is a stable mate of $i$ if there is $\mu \in S(r)$ such that $\mu(i)=j$. For each $i \in N$, we denote $\boldsymbol{i}$ 's best stable mate by $\boldsymbol{\mu}^{B}(\boldsymbol{i})$ and $\boldsymbol{i}$ 's worst stable mate by $\boldsymbol{\mu}^{W}(\boldsymbol{i})$, i.e., there are stable matchings $\mu^{\prime}, \mu^{\prime \prime} \in S(r)$ such that $\mu^{B}(i)=\mu^{\prime}(i)$ and $\mu^{W}(i)=\mu^{\prime \prime}(i)$ and for all stable matchings $\mu \in S(r)$, $r_{i}\left(\mu^{B}(i)\right) \leq r_{i}(\mu(i)) \leq r_{i}\left(\mu^{W}(i)\right)$.

Lemma 1. Let $r$ be a solvable roommate problem. The function $\mu^{B}: N \rightarrow N$ is a matching if and only if the function $\mu^{W}: N \rightarrow N$ is a matching if and only if $S(r)$ is a singleton, i.e., there is a unique stable matching at $r$.

\footnotetext{
${ }^{11}$ For each $n \in\{2,3\}$, for each solvable roommate problem $r$, and for each stable matching $\mu \in S(r)$, $\Gamma^{A}(r, \mu)=B^{1}(r)$. For $n=2$, there is a unique solvable roommate problem $r$ and for its unique stable matching $\mu \in S(r)$ we have $\Gamma^{A}(r, \mu)=B^{2}(r)$.
} 
The proof of Lemma 1 follows easily from the decomposition lemma. ${ }^{12}$

Lemma 2. [Decomposition lemma (Diamantoudi et al., 2004, Footnote 5).] ${ }^{13}$

Let $r$ be a solvable roommate problem. For each $i \in N$, if $\mu, \mu^{\prime} \in S(r)$ and agent $i$ prefers $\mu(i)$ to $\mu^{\prime}(i)$, then agent $\mu(i)$ prefers his mate at $\mu^{\prime}$ to his mate at $\mu$ and also agent $\mu^{\prime}(i)$ prefers his mate at $\mu^{\prime}$ to his mate at $\mu$, i.e., $r_{\mu(i)}\left(\mu^{\prime}(\mu(i))\right)<r_{\mu(i)}(i)$ and $r_{\mu^{\prime}(i)}(i)<r_{\mu^{\prime}(i)}\left(\mu\left(\mu^{\prime}(i)\right)\right)$.

Proof of Lemma 1. Let $r$ be a solvable roommate problem. If $S(r)$ is a singleton, then (by definition) $\mu^{B}$ and $\mu^{W}$ coincide with the unique stable matching. Now suppose $|S(r)|>$ 1. Then, for some $i \in N, \mu^{B}(i) \neq \mu^{W}(i)$. Let $\mu, \mu^{\prime} \in S(r)$ such that $\mu(i)=\mu^{B}(i)$ and $\mu^{\prime}(i)=\mu^{W}(i)$. From Lemma $2, j \equiv \mu(i)$ prefers $\mu^{\prime}(j)$ to $\mu(j)=i$. Hence, $\mu^{B}(j) \neq i$. Hence, $\mu^{B}\left(\mu^{B}(i)\right)=\mu^{B}(\mu(i))=\mu^{B}(j) \neq i$. So, $\mu^{B}$ is not a matching. Applying Lemma 2 to $\mu^{\prime}(i)$ and using similar arguments shows that $\mu^{W}$ is not a matching either.

The next lemma will be useful for the proof of Theorem 3. It says that whenever an agent obtains his best stable mate the latter obtains his worst stable mate, and vice versa. This result is related to but logically independent of the decomposition lemma. ${ }^{14}$

Lemma 3. Let $r$ be a solvable roommate problem. For each $i \in N, \mu^{W}\left(\mu^{B}(i)\right)=i$ and $\mu^{B}\left(\mu^{W}(i)\right)=i$.

Proof. Let $r$ be a solvable roommate problem. Let $i \in N$. Let $j \equiv \mu^{B}(i)$. Suppose to the contrary that $\mu^{W}(j) \neq i$. By definition of $\mu^{W}(j)$, there is $\mu \in S(r)$ such that $\mu(j)=\mu^{W}(j) \neq i$ and $r_{j}(i)<r_{j}(\mu(j))$. Moreover, by definition of $\mu^{B}(i), r_{i}(j)<r_{i}(\mu(i))$. Hence, $\{i, j\}$ blocks $\mu$, a contradiction to $\mu \in S(r)$. Hence, $\mu^{W}(j)=i$. Hence, $\mu^{W}\left(\mu^{B}(i)\right)=i$.

Next, we prove the second statement. Note that $\mu^{B}$ is injective. (To see this, suppose that there are $i, i^{\prime} \in N$ such that $\mu^{B}(i)=\mu^{B}\left(i^{\prime}\right)=j$. From the first statement it follows that $i=\mu^{W}\left(\mu^{B}(i)\right)=\mu^{W}\left(\mu^{B}\left(i^{\prime}\right)\right)=i^{\prime}$.) Since $N$ is finite and $\mu^{B}: N \rightarrow N$ is injective, $\mu^{B}$ is bijective. Let $i \in N$. There is (exactly one) $j \in N$ such that $\mu^{B}(j)=i$. From the first statement, $\mu^{W}\left(\mu^{B}(j)\right)=j$. So, $\mu^{W}(i)=j$. Substituting $j=\mu^{W}(i)$ in $\mu^{B}(j)=i$ yields the desired conclusion.

For each $i \in N$, we define agent $i$ 's rank gap in the core as $r_{i}\left(\mu^{W}(i)\right)-r_{i}\left(\mu^{B}(i)\right)$. As a first measure of the size of the core we consider the maximal rank gap in the core. Formally,

\footnotetext{
${ }^{12}$ The first version of the decomposition lemma for marriage problems appears in Knuth (1976, page 29) and is attributed to J.H. Conway. See also Roth and Sotomayor (1990, Corollary 2.21).

${ }^{13}$ Diamantoudi et al. (2004) prove the decomposition lemma for any number of agents and also for problems in which agents can be unacceptable to other agents.

${ }^{14}$ Since the functions $\mu^{B}$ and $\mu^{W}$ are in general not matchings (Lemma 1), we can only apply the decomposition lemma as follows. Let $i \in N$. Let $\mu$ and $\mu^{\prime}$ be any stable matchings such that $\mu(i)=\mu^{B}(i)$ and $\mu^{\prime}(i)=\mu^{W}(i)$. Since agent $i$ prefers $\mu^{B}(i)$ to $\mu^{W}(i)$, it follows from the decomposition lemma that agent $\mu^{W}(i)$ prefers his mate at $\mu^{\prime}$ (which is $i$ ) to his mate at $\mu$. Our Lemma 3 shows that in fact $\mu^{W}(i)$ prefers $i$ to any other stable mate, i.e., not only the mates at the matchings in the set $\left\{\mu: \mu\right.$ is stable and $\left.\mu(i)=\mu^{B}(i)\right\}$.
} 
the maximal $\boldsymbol{r}$-gap in the core of $\boldsymbol{r}$ is given by

$$
\boldsymbol{\Gamma}^{\boldsymbol{M}}(\boldsymbol{r}) \equiv \max _{i \in N} r_{i}\left(\mu^{W}(i)\right)-r_{i}\left(\mu^{B}(i)\right)
$$

Obviously, for any solvable roommate problem $r,{ }^{15}$

$$
\Gamma^{M}(r) \leq \boldsymbol{B}^{\mathbf{3}}(\boldsymbol{r}) \equiv \begin{cases}n-2 & \text { if } n \text { is even; } \\ n-3 & \text { if } n \text { is odd }\end{cases}
$$

Next, we show that for each $n \geq 2$ the bound $B^{3}$ is in fact tight.

Proposition 2. [Tightness of bound for maximal rank gap in core.]

For each $n \geq 2$, there is a solvable roommate problem $r$ such that $\Gamma^{M}(r)=B^{3}(r)$.

Proof. Let $n=2$. Then, $r_{i}(j)=1$ for all $i, j \in N$ with $i \neq j$ and at the unique stable matching $\mu$ the two agents are matched to one another. One immediately verifies that $\Gamma^{M}(r, \mu)=n-2=$ 0 .

\begin{tabular}{cccc}
\hline \hline$r_{1}$ & $r_{2}$ & $r_{3}$ & $r_{4}$ \\
\hline 2 & $3^{*}$ & 4 & $1^{*}$ \\
$4^{*}$ & 1 & 1 & 3 \\
3 & 4 & $2^{*}$ & 2 \\
\hline \hline
\end{tabular}

\begin{tabular}{ccccccc}
\hline \hline$r_{1}$ & $r_{2}$ & $r_{3}$ & $r_{4}$ & $\ldots$ & $r_{n-1}$ & $r_{n}$ \\
\hline 2 & $3^{*}$ & 4 & $5^{*}$ & $\ldots$ & $n$ & $1^{*}$ \\
$n^{*}$ & 1 & $\vdots$ & 3 & $\ldots$ & $n-2^{*}$ & $n-1$ \\
$\vdots$ & $\vdots$ & $\vdots$ & $\vdots$ & $\ldots$ & $\vdots$ & $\vdots$ \\
$\vdots$ & $\vdots$ & $2^{*}$ & $\vdots$ & $\ldots$ & $\vdots$ & $\vdots$ \\
\hline \hline
\end{tabular}

Table 5: Rankings in Proposition 2 for $n=4$ (left) and even $n \geq 6$ (right).

Let $n \geq 4$ be even. Consider any problem $(N, r)$ with $N=\{1,2, \ldots, n\}$ and $r$ such that for each agent $i \in N \backslash\{n\}, r_{i}(i+1)=1$ and $r_{n}(1)=1$ and for each agent $i \in N \backslash\{1,3\}$, $r_{i}(i-1)=2, r_{1}(n)=2$, and $r_{3}(2)=n-1$, as illustrated in Table 5 .

Let $\mu$ be the matching such that for each odd agent $i \in N, \mu(i)=i+1$ and for each even agent $i \in N, \mu(i)=i-1$ (the boxed matching in Table 5). We show that $\mu$ is stable. At $\mu$, each odd agent is matched to his most preferred agent and each even agent only is willing to block with a particular odd agent. Hence, there is no blocking pair for $\mu$. So, $\mu$ is stable. (So, in particular, $r$ is solvable.)

Let $\mu^{*}$ be the matching such that for each odd agent $i \in N \backslash\{1\}, \mu^{*}(i)=i-1$ and $\mu^{*}(1)=n$ and for each even agent $i \in N \backslash\{n\}, \mu^{*}(i)=i+1$ and $\mu^{*}(n)=1$ (the starred matching in Table 5). We show that $\mu^{*}$ is stable. At $\mu^{*}$, each even agent is matched to his

\footnotetext{
${ }^{15}$ The proof for odd $n$ is as follows. Suppose that for some solvable roommate problem $r, \Gamma^{M}(r)>n-3$. Then, $\Gamma^{M}(r)=n-2$ and there exists an agent $i$ such that $r_{i}\left(\mu^{W}(i)\right)-r_{i}\left(\mu^{B}(i)\right)=n-2$. In particular, there exists a stable matching $\mu \in S(r)$ such that $r_{i}(\mu(i))=n-1$, i.e., $i$ is matched to his least preferred agent. But then $i$ together with the lone wolf block $\mu$, which contradicts its stability.
} 
most preferred agent and each odd agent in $N \backslash\{3\}$ is only willing to block with a particular even agent. Therefore, there is no blocking pair for $\mu^{*}$. Hence, $\mu^{*}$ is stable.

Since by definition $\Gamma^{M}(r) \leq n-2$, it follows from $r_{3}\left(\mu^{W}(3)\right)-r_{3}\left(\mu^{B}(3)\right) \geq r_{3}\left(\mu^{*}(3)\right)-$ $r_{3}(\mu(3))=r_{3}(2)-r_{3}(4)=n-2$ that $\Gamma^{M}(r)=n-2=B^{3}(r)$.

Let $n \geq 3$ be odd. Consider the problem constructed for the case of $n-1$ agents. We add agent $n$ and define the ranks $r_{i}(n)=n-1$ for all $i=1,2, \ldots, n-1$ and the ranking $r_{n}$ such that for all $j=1,2, \ldots, n-1, r_{n}(j)=j$. The same arguments as for the case $n-1$ show that $\Gamma^{M}(r)=n-3=B^{3}(r)$.

Alternatively, we can measure the size of the core by averaging the rank gap between the worst and best stable mates over all agents. Formally, the average $\boldsymbol{r}$-gap in the core of $\boldsymbol{r}$ is given by

$$
\Gamma^{\boldsymbol{A}}(\boldsymbol{r}) \equiv \frac{1}{n} \sum_{i \in N}\left[r_{i}\left(\mu^{W}(i)\right)-r_{i}\left(\mu^{B}(i)\right)\right] .
$$

An immediate consequence of $\Gamma^{M}(r) \leq B^{3}(r)$ is that $\Gamma^{A}(r) \leq B^{3}(r)$. The next result provides a better bound than $B^{3}$ for the average rank gap in the core. ${ }^{16}$ More specifically, Theorem 3 shows that the average rank gap in the core can be bounded by means of the average disagreement. Hence, Observation 2 is also robust with respect to the size of the core: if rankings are sufficiently similar $\left(\Delta^{A}(r)\right.$ is small), then from each individual agent's point of view all stable matchings are close to one another $\left(\Gamma^{A}(r)\right.$ is small).

Theorem 3. [Bound for average rank gap in core.] For each solvable roommate problem $r, \Gamma^{A}(r) \leq \boldsymbol{B}^{\mathbf{4}}(\boldsymbol{r}) \equiv \Delta^{A}(r)$.

Proof. Let $r$ be a solvable roommate problem. It follows from Lemma 3 that

$$
\sum_{i \in N} r_{i}\left(\mu^{W}(i)\right)=\sum_{j \in N} r_{\mu^{B}(j)}(j) \text { and } \sum_{i \in N} r_{i}\left(\mu^{B}(i)\right)=\sum_{j \in N} r_{\mu^{W}(j)}(j) .
$$

Therefore, $\sum_{i \in N}\left[r_{i}\left(\mu^{W}(i)\right)-r_{i}\left(\mu^{B}(i)\right)\right]=\sum_{j \in N}\left[r_{\mu^{B}(j)}(j)-r_{\mu^{W}(j)}(j)\right]$. Then, since

$$
\sum_{j \in N}\left[r_{\mu^{B}(j)}(j)-r_{\mu^{W}(j)}(j)\right] \leq \sum_{j \in N}\left[\max _{i \in N \backslash\{j\}} r_{i}(j)-\min _{i \in N \backslash\{j\}} r_{i}(j)\right]=\sum_{j \in N} \delta^{r}(j),
$$

it follows that $\Gamma^{A}(r)=\frac{1}{n} \sum_{i \in N}\left[r_{i}\left(\mu^{W}(i)\right)-r_{i}\left(\mu^{B}(i)\right)\right] \leq \frac{1}{n} \sum_{i \in N} \delta^{r}(i)=\Delta^{A}(r)$.

Our final result shows that the bound $B^{4}$ is "essentially tight." More precisely, for each population size $n$ that is a multiple of 6 , we construct a solvable roommate problem $r$ with $n$ agents such that $\Gamma^{A}(r)=B^{4}(r)$.

\footnotetext{
${ }^{16}$ One immediately verifies that for each solvable roommate problem $r, B^{4}(r) \leq B^{3}(r)$.
} 
Proposition 3. ["Essential" tightness of bound $B^{4}$ for average rank gap in core.]

For each $n=6 l$ where $l$ is a positive integer, there is a solvable roommate problem $r$ such that

$$
\Gamma^{A}(r)=B^{4}(r)
$$

Proof. Let $n=6 l$ where $l$ is a positive integer. We construct a problem $(N, r)$ such that $N=\{1,2, \ldots, n\}$ with $n=6 l$. Table 6 illustrates the construction for the case where $n=12$, i.e., $l=2$. We impose the following restrictions on the rankings of $N$ :

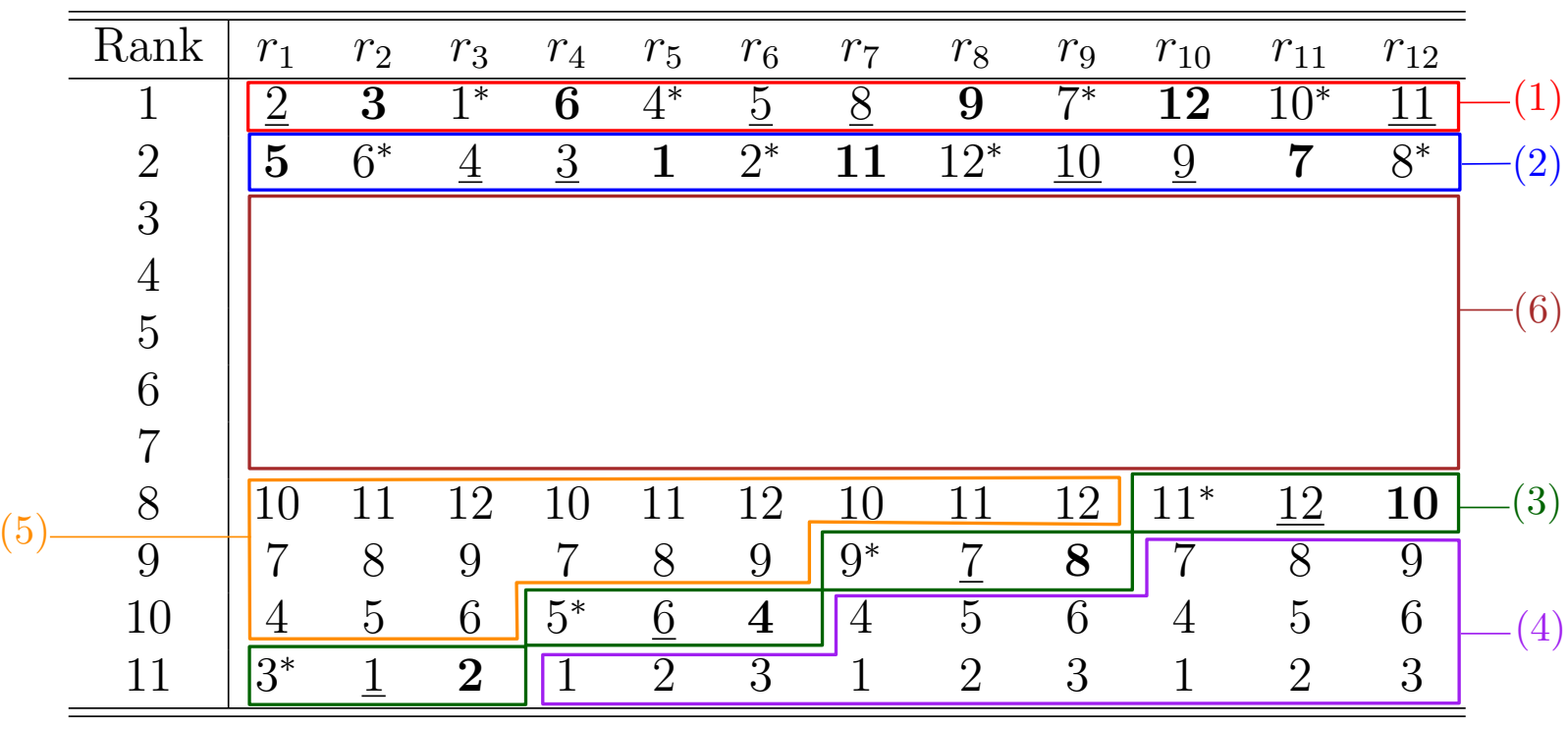

Table 6: Rankings in Proposition 3 for $n=12$. Matchings $\mu^{1}$ (underlined), $\mu^{2}$ (starred), and $\mu^{3}$ (boldfaced).

(1) For each $k \in\{0,1, \ldots, l-1\}$ and each $i \in\{1,2\}$, we have $r_{i+6 k}(i+6 k+1)=1$. For each $k \in\{0,1, \ldots, l-1\}$ and each $i \in\{5,6\}$, we have $r_{i+6 k}(i+6 k-1)=1, r_{3+6 k}(3+6 k-2)=1$, and $r_{4+6 k}(4+6 k+2)=1$.

(2) For each $k \in\{0,1, \ldots, l-1\}$ and each $i \in\{1,2\}$, we have $r_{i+6 k}(i+6 k+4)=2$. For each $k \in\{0,1, \ldots, l-1\}$ and each $i \in\{5,6\}$, we have $r_{i+6 k}(i+6 k-4)=2, r_{3+6 k}(3+6 k+1)=2$, and $r_{4+6 k}(4+6 k-1)=2$.

(3) For each $k \in\{0,1, \ldots, l-1\}$ and each $i \in\{2,3\}$, we have $r_{i+6 k}(i+6 k-1)=n-2 k-1$. For each $k \in\{0,1, \ldots, l-1\}$ and each $i \in\{4,5\}$, we have $r_{i+6 k}(i+6 k+1)=n-2 k-2$, $r_{1+6 k}(1+6 k+2)=n-2 k-1$, and $r_{6+6 k}(6+6 k-2)=n-2 k-2$.

(4) For each $i \in\{1,2,3\}$, each $p \in\{1,2, \ldots, 2 l-1\}$, and each $k \in\{0,1, \ldots, p-1\}$, we have $r_{i+3 p}(i+3 k)=n-k-1$.

(5) For each $i \in\{1,2,3\}$, each $p \in\{0,1, \ldots, l\}$, and each $k \in\{p+1, \ldots, 2 l-1\}$, we have $r_{i+3 p}(i+3 k)=n-k-1$. 
(6) Each agent $i \in N$ places the agents in $N \backslash\{i\}$ that have not been assigned yet to a rank in arbitrary order (so that they get ranks 3 up to $n-2 l-1$ ).

First, we show that the rankings are well-defined i.e., there is no incompatibility in the construction above. In (1), we describe the first row of the table and in (2), the second row of the table. Note that for each agent's ranking, the agent in row 1 differs from the one in row 2. In (3), we describe a threshold row for each agent which will represent his largest rank at a stable matching. Note that for each $s \in\{0,1, \ldots, 2 l-1\}$ and for each agent $i \in\{1+3 s, 2+3 s, 3+3 s\}$, the agent in the threshold row is again an agent in the set $\{1+3 s, 2+3 s, 3+3 s\}$ and has not appeared in the construction before (i.e., (1) and (2)). In (4), we describe the ranks below the threshold row for each agent. Note that for each agent $i$, these ranks are only for certain agents $j$ with $j<i$ (and they have not appeared before). In (5), we describe some ranks above the threshold row for each agent. Note that for each agent $i$, these ranks are only for certain agents $j$ with $j>i$ (and they have not appeared before). Conditions (4) and (5) are symmetric in the sense that for any two agents $i, j \in N$, agent $i$ has agent $j$ in some rank described by (4) if and only if agent $j$ has agent $i$ in some rank described by (5).

Next, we define three matchings $\mu^{1}, \mu^{2}$, and $\mu^{3}$. Let $\mu^{1}$ be the matching such that for each odd agent $i \in N, \mu^{1}(i)=i+1$ (the underlined matching in Table 6 ). Let $\mu^{2}$ be the matching such that for each $k \in\{0,1, \ldots, l-1\}, \mu^{2}(1+6 k)=3+6 k, \mu^{2}(2+6 k)=6+6 k$, and $\mu^{2}(4+6 k)=5+6 k$ (the starred matching in Table 6). Let $\mu^{3}$ be the matching such that for each $k \in\{0,1, \ldots, l-1\}, \mu^{3}(1+6 k)=5+6 k, \mu^{3}(2+6 k)=3+6 k$, and $\mu^{3}(4+6 k)=6+6 k$ (the boldfaced matching in Table 6 ). We show that $\mu^{1}$ is stable. Because of the symmetry in our construction, the stability of $\mu^{2}$ and $\mu^{3}$ follows from similar arguments. For each $k \in\{0,1, \ldots, l-1\}$, we have

$$
\begin{aligned}
& r_{1+6 k}\left(\mu^{1}(1+6 k)\right)=r_{1+6 k}(1+6 k+1)=1 \text { since } 1+6 k \text { is odd and }(1), \\
& r_{2+6 k}\left(\mu^{1}(2+6 k)\right)=r_{2+6 k}(2+6 k-1)=n-2 k-1 \text { since } 2+6 k \text { is even and }(3), \\
& r_{3+6 k}\left(\mu^{1}(3+6 k)\right)=r_{3+6 k}(3+6 k+1)=2 \text { since } 3+6 k \text { is odd and }(2), \\
& r_{4+6 k}\left(\mu^{1}(4+6 k)\right)=r_{4+6 k}(4+6 k-1)=2 \text { since } 4+6 k \text { is even and }(2), \\
& r_{5+6 k}\left(\mu^{1}(5+6 k)\right)=r_{5+6 k}(5+6 k+1)=n-2 k-2 \text { since } 5+6 k \text { is odd and }(3), \text { and } \\
& r_{6+6 k}\left(\mu^{1}(6+6 k)\right)=r_{6+6 k}(6+6 k-1)=1 \text { since } 6+6 k \text { is even and }(1) .
\end{aligned}
$$

At $\mu^{1}$, for each $k \in\{0,1, \ldots, l-1\}$, agents $1+6 k$ and $6+6 k$ are matched to their most preferred agent. For each $k \in\{0,1, \ldots, l-1\}$, agents $3+6 k$ and $4+6 k$ are only willing to block with particular agents, namely $1+6 k$ and $6+6 k$, respectively, but as we have just noticed each of the latter agents is matched to his most preferred agent. It only remains to prove that there is no blocking pair contained in the set $\{2+6 k: k=0,1, \ldots, l-1\} \cup\{5+6 k: k=0,1, \ldots, l-1\}$. First, for each $k, k^{\prime} \in\{0,1, \ldots, l-1\}$ such that $k^{\prime}<k$, we have 


$$
\begin{gathered}
r_{2+6 k}\left(2+6 k^{\prime}\right)=r_{2+6 k}\left(2+3\left(2 k^{\prime}\right)\right) \stackrel{\text { by (4) }}{=} n-2 k^{\prime}-1>n-2 k-1=r_{2+6 k}\left(\mu^{1}(2+6 k)\right), \\
r_{5+6 k}\left(5+6 k^{\prime}\right)=r_{5+6 k}\left(2+3\left(1+2 k^{\prime}\right)\right) \stackrel{\text { by (4) }}{=} n-2 k^{\prime}-2>n-2 k-2=r_{5+6 k}\left(\mu^{1}(5+6 k)\right), \text { and } \\
r_{2+6 k}\left(5+6 k^{\prime}\right)=r_{2+6 k}\left(2+3\left(1+2 k^{\prime}\right)\right) \stackrel{\text { by (4) }}{=} n-2 k^{\prime}-2>n-2 k-1=r_{2+6 k}\left(\mu^{1}(2+6 k)\right) .
\end{gathered}
$$

Second, for each $k, k^{\prime} \in\{0,1, \ldots, l-1\}$ such that $k^{\prime} \leq k$, we have

$$
r_{5+6 k}\left(2+6 k^{\prime}\right)=r_{5+6 k}\left(2+3\left(2 k^{\prime}\right)\right) \stackrel{\text { by }(4)}{=} n-2 k^{\prime}-1>n-2 k-2=r_{5+6 k}\left(\mu^{1}(5+6 k)\right) .
$$

Hence, there is no blocking pair for $\mu^{1}$. So, $\mu^{1}$ is stable. (So, in particular, $r$ is solvable.)

Finally, we prove that $\Gamma^{A}(r)=B^{4}(r)$. We first calculate for each $i \in N, \delta^{r}(i)$, to obtain $\sum_{i \in N} \delta^{r}(i)$. By (1), for each $i \in N, \min _{j \in N \backslash\{i\}} r_{j}(i)=1$. By (3), (4), and (5), for each $s \in\{0,1, \ldots, 2 l-1\}$ and each $i \in\{1+3 s, 2+3 s, 3+3 s\}$, we have $\max _{j \in N \backslash\{i\}} r_{j}(i)=n-s-1$. Then,

$$
\begin{aligned}
\sum_{i \in N} \delta^{r}(i) & =\sum_{s \in\{0, \ldots, 2 l-1\}} \sum_{j \in\{1,2,3\}} \delta^{r}(j+3 s) \\
& =\sum_{s \in\{0, \ldots, 2 l-1\}} \sum_{j \in\{1,2,3\}}[(n-s-1)-1]=\sum_{s \in\{0, \ldots, 2 l-1\}} \sum_{j \in\{1,2,3\}}(n-s-2) \\
& =\sum_{s \in\{0, \ldots, 2 l-1\}} 3(n-s-2)=6 l(n-2)-\sum_{s \in\{0, \ldots, 2 l-1\}} 3 s \\
& =6 l(6 l-2)-\frac{3(2 l-1) 2 l}{2}=30 l^{2}-9 l .
\end{aligned}
$$

Next, we consider $\sum_{i \in N}\left[r_{i}\left(\mu^{W}(i)\right)-r_{i}\left(\mu^{B}(i)\right)\right]$. For each $i \in N$, let $W_{i}$ and $B_{i}$ be the worst mate and the best mate in $\left\{\mu^{1}(i), \mu^{2}(i), \mu^{3}(i)\right\}$, respectively. For each $k \in\{0,1, \ldots, l-1\}$ and each $j \in\{1,2,3\}$, we have

$$
r_{j+6 k}\left(W_{j+6 k}\right)-r_{j+6 k}\left(B_{j+6 k}\right)=(n-2 k-1)-1=n-2 k-2 .
$$

Similarly, for each $k \in\{0,1, \ldots, l-1\}$ and each $j \in\{4,5,6\}$, we have

$$
r_{j+6 k}\left(W_{j+6 k}\right)-r_{j+6 k}\left(B_{j+6 k}\right)=(n-2 k-2)-1=n-2 k-3 .
$$

Hence,

$$
\begin{aligned}
\sum_{i \in N}\left[r_{i}\left(\mu^{W}(i)\right)-r_{i}\left(\mu^{B}(i)\right)\right] & \geq \sum_{i \in N}\left[r_{i}\left(W_{i}\right)-r_{i}\left(B_{i}\right)\right] \\
& =\sum_{k \in\{0, \ldots, l-1\}} \sum_{j \in\{1, \ldots, 6\}}\left[r_{j+6 k}\left(W_{j+6 k}\right)-r_{j+6 k}\left(B_{j+6 k}\right)\right] \\
& =\sum_{k \in\{0, \ldots, l-1\}} \sum_{j \in\{1,2,3\}}(n-2 k-2)+\sum_{k \in\{0, \ldots, l-1\}} \sum_{j \in\{4,5,6\}}(n-2 k-3) \\
& =\sum_{k \in\{0, \ldots, l-1\}} 3(n-2 k-2+n-2 k-3)
\end{aligned}
$$




$$
\begin{aligned}
& =\sum_{k \in\{0, \ldots, l-1\}} 3(2 n-4 k-5) \\
& =3 l(2 n-5)-\sum_{k \in\{0, \ldots, l-1\}} 12 k \\
& =36 l^{2}-15 l-\frac{12 l(l-1)}{2} \\
& =30 l^{2}-9 l .
\end{aligned}
$$

From Theorem 3 and $\sum_{i \in N}\left[r_{i}\left(\mu^{W}(i)\right)-r_{i}\left(\mu^{B}(i)\right)\right] \geq \sum_{i \in N} \delta^{r}(i)$, it follows that for each $i \in N, W_{i}=\mu^{W}(i)$ and $B_{i}=\mu^{B}(i)$, and more importantly,

$$
\Gamma^{A}(r)=\frac{1}{n} \sum_{i \in N}\left[r_{i}\left(\mu^{W}(i)\right)-r_{i}\left(\mu^{B}(i)\right)\right]=5 l-\frac{3}{2}=\frac{1}{n} \sum_{i \in N} \delta^{r}(i)=B^{4}(r) .
$$

\section{Concluding remarks}

As we have mentioned in the Introduction, our paper is closely related to Holzman and Samet (2014). Holzman and Samet (2014) study marriage problems which constitute a particular kind of roommate problems: the difference is that in a marriage problem agents are either male or female, and a man (woman) only wants to be matched to a woman (man) or to him(her)self. ${ }^{17}$ Below we first discuss the main assumptions in our study and Holzman and Samet (2014). Next, we will describe the main differences in the results and proofs to show that our study is not a straightforward adaptation of Holzman and Samet's (2014) analysis. Finally, we will discuss some ideas for possible future research.

Our study and Holzman and Samet (2014) make the following assumptions. First, Holzman and Samet (2014) assume that the number of men equals the number of women. We do not make the corresponding assumption on the number of agents in our roommate problems: the number of agents need not be even. Second, Holzman and Samet (2014) assume that each man is acceptable to all women and that each woman is acceptable to all men. A parallel assumption in our study is that all agents are mutually acceptable. This is a restrictive but not unusual assumption (see, e.g., Irving, 1985 and Pittel, 1993). More importantly, in certain situations the assumption seems very natural. For instance, when pairing police officers on patrols or assigning students to share double rooms in dorms, the involved agents may prefer any co-worker or roommate (instead of losing a job, living too far from campus, or paying an unfeasible rent). In these applications, all agents are mutually acceptable. Suppose we drop the assumption. Then, it can happen that some agents find some other agent unacceptable. How should we (re)define or interpret the concepts and tools employed in our study?

It would still be possible (mathematically) to continue to use the same definitions of the maximal and average rank gap between mates and the maximal and average rank gap in the

\footnotetext{
${ }^{17}$ In other words, each man (woman) puts any other men (women) below the outside option of being single.
} 
core. However, the interpretation of rank gaps would become less clear. The reason is that, as mentioned in the Introduction, we implicitly assume that rank gaps have a similar meaning for different agents. This seems reasonable for "non-extreme" situations where variations of preference intensities are limited. However, as soon as agents can be unacceptable, it is clear that preference intensities need no longer be limited. Dropping the assumption of mutual acceptability also complicates the notion and interpretation of maximal and average disagreements in at least two ways. First, suppose agent $i$ is agent $j$ 's most preferred mate, while agent $k$ finds agent $i$ unacceptable. How should we quantify the disagreement over agent $i$ ? We believe there is not an obvious answer to this question. Second, suppose some agent $i$ finds all other agents unacceptable, while $j$ finds $i$ acceptable and $k$ finds $i$ unacceptable. Should we remove agent $i$ from the market? If so, how should we adjust the rankings of the other agents after the removal of agent $i$ ? Again, we do not have a clear answer. In short, dropping the assumption of mutual acceptability leads to conceptual problems. Interestingly, these problems disappear if unacceptability is allowed in a structural way, which is exactly the case of Holzman and Samet (2014)! In their study there is an exogenously given partition of men and women and it is assumed that each man is acceptable to all women (but not to any of the other men) and that each woman is acceptable to all men (but not to any of the other women).

Next, we compare the tools, results, and proofs in Holzman and Samet (2014) and our study. We have adapted their tools for our roommate problems. Rank gaps between mates are defined in exactly the same way. The size of the core is measured differently: while we consider the average and maximal rank gap in the core, Holzman and Samet (2014) only consider two (separate) average metrics: a woman-metric and a man-metric. Because of the bilaterality of the markets in Holzman and Samet (2014), they measure disagreement (or displacement) of an agent with respect to the rankings of the agents on the other side of the market. We measure disagreement with respect to all other agents. Even in roommate problems derived from a common ordering as in Observation 2, there is still a lot of disagreement. In contrast, marriage problems where all men order the women in the same way and where all women order the men in the same way, have zero disagreement. The difference is due to the somewhat artificial disagreement that arises as agents have to rank themselves last in our roommate problems: if all other agents rank $i$ highest, then $i$ still has to rank some other agent $j$ highest, so there is disagreement over $j$. This makes our bound $B^{2}$ even more conservative than its marriage counterpart (Holzman and Samet, 2014, Theorem 2). ${ }^{18}$

Holzman and Samet (2014) study rank gaps at stable matchings and the size of the core of marriage problems, and provide upper bounds that are "essentially" tight. The particular class of marriage problems studied in Holzman and Samet (2014) is not a subclass of the class of roommate problems that we study. The reason is again that we assume that all agents

\footnotetext{
${ }^{18}$ Holzman and Samet (2014, p.283) conjecture that the marriage counterpart of $B^{2}$ can be reduced significantly.
} 
are mutually acceptable. Obviously, our class of (one-sided) roommate problems is also not a subclass of the class of (two-sided) marriage problems. Therefore, our results and those of Holzman and Samet (2014) are not logically related. However, the proofs of our Theorems 1 and 2 are similar to those of Theorems 1 and 2 in Holzman and Samet (2014). All of our other results and proofs (including Proposition 1, Lemma 3, Proposition 2, Theorem 3, and Proposition 3) are novel. Our propositions show the tightness of our bounds. The proofs of Propositions 1 and 3 are based on a very non-trivial construction of particular roommate markets. There are (at least) three reasons that explain the non-triviality of the construction: 1) the existence of stable matchings is not guaranteed for roommate markets, 2) we cannot use "universal rankings" (as in Example 1 of Holzman and Samet, 2014), and 3) there are no side-optimal or side-pessimal stable matchings (as in Example 3 of Holzman and Samet, 2014). The (non-trivial) proof of Theorem 3 is based on Lemma 3, which is interestingly related to but logically independent of the decomposition lemma.

We have provided tightness results for the bounds of the maximal rank gap between mates and the maximal/average rank gap in the core (Propositions 1, 2, and 3). In the proofs of these results we exhibit and construct roommate problems such that a particular measure coincides with its bound. In each of these cases, it is not difficult to see that certain (combinations of) changes in the rankings can be made such that for the resulting rankings the measure again coincides with its bound. A natural research question would be to determine (for a given population size) the proportion of roommate problems for which the measure coincides with the upper bound. ${ }^{19}$ However, given a tight problem (as in the proofs of Propositions 1 , 2 , and 3) it does not seem unlikely that some "permitted" combinations of changes may be quite radical, which might make it complicated to find all of them. Also, exhaustive computations by computer or even simulations do not seem a feasible alternative approach as the number of possible problems for size $n$ is $((n-1) !)^{n}$ which grows exponentially in $n$ (for instance, for $n=6$ and $n=12$ there are already $2.99 \mathrm{e}+12$ and $1.64 \mathrm{e}+91$ problems, respectively). More generally, it would be interesting to obtain the distribution of the distance between the measure and its upper bound (not only the probability mass at 0). Again, such an analysis seems highly complicated but could be a very ambitious future research line.

\section{References}

[1] J. Alcalde (1994). Exchange-Proofness or Divorce-Proofness? Stability in One-Sided Matching Markets. Economic Design 1(1): 275-287.

[2] A. Bogomolnaia and M.O. Jackson (2002). The Stability of Hedonic Coalition Structures. Games and Economic Behavior 38(2): 201-230.

\footnotetext{
${ }^{19}$ We thank a reviewer for posing this question.
} 
[3] K.-S. Chung (2000). On the Existence of Stable Roommate Matchings. Games and Economic Behavior 33(2): 206-230.

[4] G. Demange and M. Wooders (2004). Group Formation in Economics: Networks, Clubs and Coalitions. Cambridge University Press, Cambridge.

[5] E. Diamantoudi, E. Miyagawa, and L. Xue (2004). Random Paths to Stability in the Roommate Problem. Games and Economic Behavior 48(1): 18-28.

[6] D. Gale and L.S. Shapley (1962). College Admissions and the Stability of Marriage. American Mathematical Monthly 69(1): 9-15.

[7] J. Gudmundsson (2014). When do Stable Roommate Matchings Exist? A Review. Review of Economic Design 18(2): 151-161.

[8] D. Gusfield and R. Irving (1989). The Stable Marriage Problem: Structure and Algorithms. MIT Press, Boston.

[9] R. Holzman and D. Samet (2014). Matching of Like Rank and the Size of the Core in the Marriage Problem. Games and Economic Behavior 88: 277-285.

[10] R.W. Irving (1985). An Efficient Algorithm for the Stable Roommates Problem. Journal of Algorithms, 6, 577-595.

[11] M.O. Jackson (2008). Social and Economic Networks. Princeton University Press, Princeton.

[12] M.O. Jackson and A. Watts (2002). The Evolution of Social and Economic Networks. Journal of Economic Theory 106(2): 265-295.

[13] B. Klaus and F. Klijn (2010). Smith and Rawls Share a Room: Stability and Medians. Social Choice and Welfare 35(4): 647-667.

[14] D.E. Knuth (1976). Mariages Stables et leurs Relations avec d'autres Problèmes Combinatoires. Les Presses de l'Université de Montréal, Montréal.

[15] D. Manlove (2013). Algorithmics of Matching under Preferences. Series on Theoretical Computer Science: Volume 2. World Scientific Publishing.

[16] B. Pittel (1993). The "Stable Roommates" Problem with Random Preferences. Annals of Probability 21(3): 1441-1477.

[17] A.E. Roth and M.A.O. Sotomayor (1990). Two-Sided Matching: A Study in GameTheoretic Modeling and Analysis. Econometric Society Monograph Series. New York: Cambridge University Press. 
[18] J. Tan (1991). A Necessary and Sufficient Condition for the Existence of a Complete Stable Matching. Journal of Algorithms 12(1): 154-178. 